\title{
Pediatric Hemophagocytic Syndromes: A Diagnostic and Therapeutic Challenge
}

\author{
Nada Jabado, MD, PhD; Christine McCusker, MD; \\ Genevieve de Saint Basile, MD, PhD
}

\begin{abstract}
Pediatric hemophagocytic syndrome (HS) is a severe and often fatal clinical disorder. This syndrome is frequently unrecognized, and thus, affected children may receive suboptimal management, leading to an increase in mortality. The purpose of this review is to provide a clinical guide to (1) the recognition of $\mathrm{HS}$ based on clinical, biologic, and pathologic features; (2) the identification of the primary cause of HS in a given affected child; and (3) the initiation of effective treatment in a timely manner.
\end{abstract}

\section{Background}

Pediatric hemophagocytic syndrome (HS) is a distinct clinical entity in which excessive uncontrolled activation and proliferation of $\mathrm{T}$ cells and macrophages occur and are often fatal. First described in 1939 by Scott and Robb-Smith as a histiocytic reticulosis, a neoplastic proliferation of histiocytes, ${ }^{1}$ this syndrome has since then been given several other denominations, including

N. Jabado - Division of Haematology and Oncology, Department of Paediatrics, Montreal Children's Hospital, McGill University Health Centre, Montreal, Quebec; C. McCusker - Division of Allergy and Immunology, Department of Paediatrics, Montreal Children's Hospital, McGill University Health Centre, Montreal, Quebec; G. de Saint Basile - INSERM U429, Hôpital Necker Enfants-Malades, 149 rue de Sèvres, 75015 Paris, France

Correspondence to: Dr. Nada Jabado, Division of Haematology and Oncology, Department of Paediatrics, Montreal Children's Hospital, McGill University Health Centre, Montreal, PQ H3Z 2Z3; E-mail:

nada.jabado@mcgill.ca

N. Jabado and C. McCusker are recipients of a "Chercheur Boursier" Award from Fondation de la Recherche en Sante au Québec hemophagocytic histiocytosis, histiocytic disorder, macrophage activation syndrome, and reactive hemophagocytic lymphohistiocytosis (HLH). ${ }^{2,3}$ To date, this syndrome remains ill-recognized in children, leading to false or delayed diagnosis and suboptimal management. Etiologically, HS is a component of several inherited disorders in which it is present at onset or during the course of the disease. It has also been associated with a variety of viral, bacterial, fungal, and parasitic infections, as well as with collagen-vascular diseases ${ }^{4-6}$ and malignancies, particularly T-cell malignancies. ${ }^{7}$ The association between HS and infection is important because both sporadic and familial cases of HS are often precipitated by acute infections; HS mimics overwhelming infectious sepsis, misleading diagnosis, ${ }^{8}$ and may obscure the diagnosis of precipitating treatable infectious illnesses, including visceral leishmaniasis and tuberculosis. ${ }^{9-12}$ The diversity of diseases associated with HS and its strong link with intracellular infections have led to delays in determining etiology and initiating proper care. In recent years, our knowledge of the common pathogenic mechanisms underlying this disorder has dramatically improved, and the terminology 
and classification of disorders associated with HS are under revision. This review aims to provide clinicians with

1. a definition of HS as a clinical and biologic entity that will help with the recognition of this syndrome in an affected child and the initiation of proper management;

2. a classification of potential diseases leading to HS, based on our current knowledge of their molecular defects and providing the current means of establishing a molecular diagnosis; and

3. a brief overview of available treatment options, based on our understanding of disease mechanisms.

\section{Recognizing HS}

\section{Etiopathogenesis}

In response to infection, innate and adaptive elements of the immune system act in concert to clear the pathogen and generate memory cells of adaptive immunity. ${ }^{13,14}$ In a physiologic (normal) situation, triggering of the immune system by an intracellular organism leads to transient activation and expansion of the lymphohistiocytic compartment. Transient production of interferon- $\gamma$ (INF- $\gamma$ ) leads to transient expansion and activation of both the lymphocyte and macrophage compartments. The intensity of the immune response depends on the type of infecting antigen, its structure, dose, localization, and duration of infection in the host. ${ }^{15}$ Once the initial infection has been cleared, control of the response in normal individuals results in contraction of the immune system and a return to baseline for both lymphoid and macrophage lineages, with generation of a few memory $\mathrm{T}$ and $\mathrm{B}$ cells (Figure 1A). Homeostasis of the immune system is impaired in diseases that lead to HS. Whether the underlying primary defect is in the lymphocyte or in the macrophage compartment, uncontrolled expansion and activation of mostly $\mathrm{CD}^{+}$lymphocytes and macrophages occur, leading to an unending positive feedback loop on both cell lineages. T cells continuously produce INF- $\gamma$ and tumour necrosis factor- $\alpha$ (TNF- $\alpha$ ), which in turn continuously activate and induce the proliferation of $\mathrm{T}$ cells and activate macrophages. Activated macrophages expand and infiltrate the reticuloendothelial tissues (including bone marrow, liver, spleen, and lymph nodes, which can result in organomegaly) ${ }^{3}$ and the perivascular structures of the brain, inducing central nervous system (CNS) involvement. ${ }^{16-18}$ These activated macrophages avidly phagocytose all nearby hematopoietic lineages, including red blood cells (hence the term "hemophagocytosis"), granulocytes, and platelets (see Figure 1B). They produce cytokines, including interleukin (IL)- 1, TNF- $\alpha$, and IL-6. ${ }^{19}$ High levels and prolonged production of these cytokines result in fever, hemodilution with hyponatremia, hypertriglyceridemia, and coagulation abnormalities. Also, oversecretion of IL-18 by monocytes in patients with HS has been described ${ }^{20}$ and may further enhance TNF$\alpha$ and IFN- $\gamma$ production by $\mathrm{T}$ lymphocytes and natural killer (NK) cells as well as induce Fas ligand expression on lymphocytes, enhancing their cytotoxic effect. Increased serum levels of soluble Fas ligand, which can trigger apoptosis in such Fas-expressing tissues as the kidney, liver, and heart, are also seen in HS and may result in organ failure through increased apoptosis of cells in these tissues. ${ }^{21}$

In summary, HS results from the failure of down-regulating and limiting a $\mathrm{T}$ helper 1 (Th1)-type immune response after it is triggered. This may occur, as detailed below, through intrinsic cytotoxic T-cell and NK-cell dysfunction in patients such as is seen in hereditary forms or in rheumatoid arthritis, impairing the host ability to control underlying infectious triggers; or, alternately, it may occur through ongoing stimulation of a Th1 immune response that drives a continued expansion of the immune reaction, such as is seen in persistent infection or in malignancies.

\section{The Cytotoxic Granule-Mediated Cell Death Pathway}

The molecular characterization of several inherited disorders leading to $\mathrm{HS}$ in the past 5 years has 


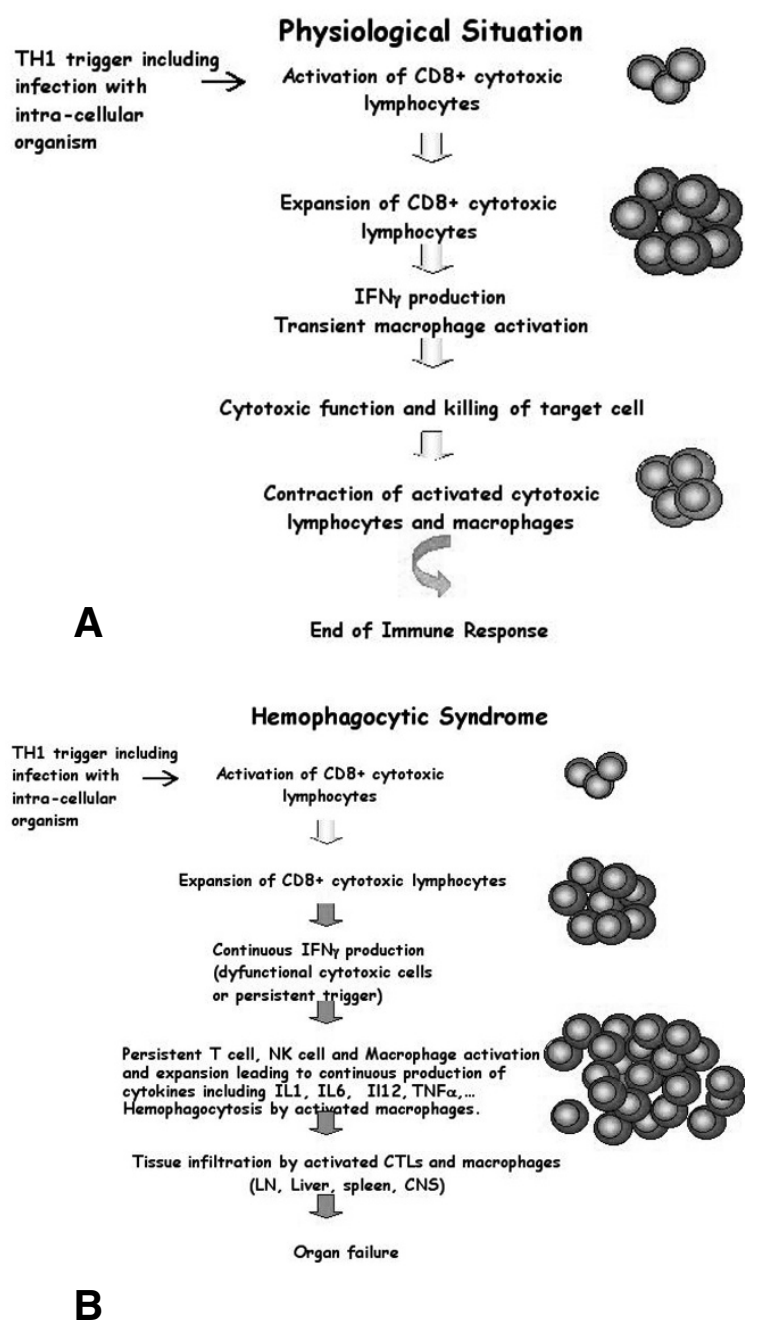

Figure 1 Schematic overview of antigen specific CD8+ T-cell response in a normal individual $(A)$ and in a patient with hemophagocytic syndrome $(B)$. In response to an infectious trigger, antigen-specific $\mathrm{CD} 8^{+}$ $\mathrm{T}$ cells transiently undergo massive expansion, use cell-mediated cytolysis, and produce interferon- $\gamma$ (IFN- $\gamma$ ). After pathogen clearance, this immune response is self-limiting and most cells die, leaving a reduced number of memory T and B cells. During the course of hemophagocytic syndrome, uncontrolled expansion of antigen-specific effectors occurs. Activated lymphocytes secrete high levels of INF- $\gamma$ and induce a feedback loop on macrophage and T cells, which continuously activate each other and expand. High levels of inflammatory cytokines are secreted, including IFN $\gamma$, tumour necrosis factor- $\alpha$, interleukin (IL)- 1, IL-6, and IL-18. Activated macrophages phagocytose bystander hematopoietic cells (hemophagocytosis). Activated lymphocytes and macrophages infiltrate various organs, resulting in massive tissue necrosis and organ failure. revolutionized our understanding of HS. Genes associated with inherited forms of $\mathrm{HS}$ are part of the cytotoxic granule-mediated cell death pathway and shed light on a previously unsuspected role for this pathway in lymphocyte homeostasis. ${ }^{13}$

The granule exocytosis cytotoxic pathway is a rapid, powerful, and iterative mechanism adapted to the killing of infected cells..$^{1322-24}$ Cytotoxic T lymphocytes (CTLs) and NK cells contain cytoplasmic lysosomes that can undergo regulated secretion in response to external stimuli. These lysosomes contain perforin (the central protein for CTL-mediated killing), granzyme, and other granule components. In resting CTLs, these cytotoxic granules move back and forth along microtubules by means of kinesin- and dynein-based motors but often cluster around the microtubule organizing centre (MTOC) in the absence of external stimuli (Figure 2A). Granule secretion is triggered by the recognition of a target cell via the T-cell receptor and/or other receptors yet to be identified at the plasma membrane of the CTLs and NK cells. Within the CTL, the MTOC moves from a perinuclear region to the contact site, repolarizing the microtubule network toward the target cell within minutes of target cell recognition. Granules migrate along microtubules to the area of cell contact in a coordinated process and fuse with the plasma cell membrane, creating an immunologic synapse (Figure 3; see also Figure 2A). Their components are secreted into the intracellular junction, and perforin and granzyme cooperate to mediate apoptosis of the target cell within 5 minutes of receptor engagement. Not all granules are exocytosed, and the remaining granules are ready for new target interaction and killing. The immunologic synapse is a distinct topologic re-arrangement of cell surface proteins formed by a ring of adhesion proteins (leukocyte function-associated antigen 1 and talin) surrounding a central domain containing a patch of signalling proteins and a distinct secretory domain in which granule exocytosis occurs.

The fact that all hereditary forms of HS have defects of cytotoxic T- and NK-cell function strongly suggests that dysfunction of this subset of lymphocytes likely plays a key role in all forms 


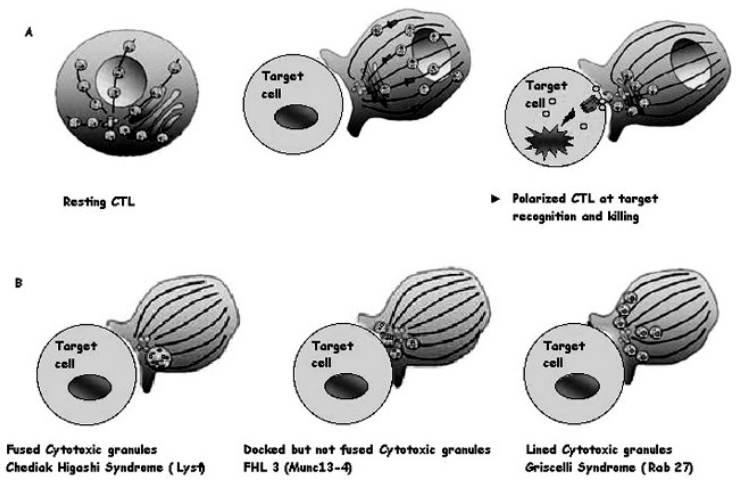

Figure 2 Cytotoxic granules in wild-type cytotoxic T lymphocytes (CTLs) and in CTLs from patients with genetic defects. A, Illustrations of the distribution of cytotoxic granules on microtubules (lines) in a resting human CTL (left panel). Perforin and granzyme are represented as red and green circles inside granules; one granule of each only is shown for clarity. After a CTL encounters a target cell, cytotoxic granules polarize and move along microtubules (middle panel) to the microtubule organizing centre (in blue), which migrates to the immunologic synapse and induces apoptosis of the target cell after the endocytosis of cytotoxic granules in its cytoplasm (right panel). $B$, Illustration of images of CTLs from patients lacking Lyst (Chédiak-Higashi syndrome), MUNC13-4 (FHL3), or RAB27A (Griscelli syndrome 2) conjugated with target cells.

of HS, whether they are acquired or inherited. Important, the hereditary forms clearly show us that $T$ cells and NK cells are the trigger for $H S$, and gaining better control of T- and NK-cell activation is the best way to manage and control the disease.

\section{Clinical, Biologic, and Pathologic Features}

The clinical presentation of HS is generally acute and dramatic (Table 1). Typically, patients become acutely ill with the sudden onset of a high and unremitting fever. Splenomegaly is the second most common clinical finding and can be associated with hepatomegaly, lymphadenopathies, jaundice, and CNS symptoms including confusion, seizures, and (more rarely) focal deficits. A maculopapular skin rash and abdominal distension have also been described. These clinical findings are suggestive of acute viral infections such as Epstein-Barr virus (EBV) infection,

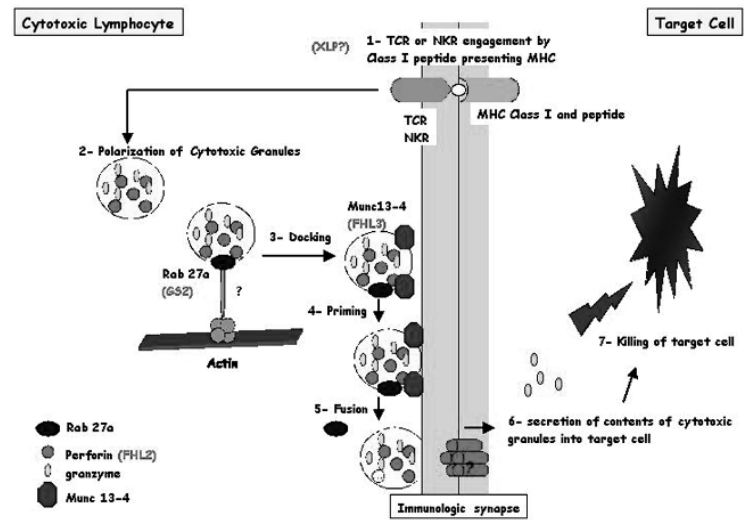

Figure 3 Schematic representation of cytotoxic granule exocytosis and target killing following target recognition by cytotoxic T lymphocytes (CTLs) or natural killer (NK) cells) Recognition of a peptide-major histocompatibility complex class I molecule presented by a target cell induces activation of cytotoxic lymphocytes (CTLs and NK cells). After cell conjugate formation, activated lymphocytes polarize their lytic granules toward the cell-to-cell contact, organized as an immunologic synapse. RAB27A is expected to promote the terminal transport and/or the docking step of the cytotoxic granules at the immunologic synapse. For its function, RAB27A potentially associates with unknown effectors and with MUNC13-4. MUNC13-4 functions as a priming factor, allowing cytotoxic granules to reach a fusion-competent state before membrane fusion and granule secretion occur. In $30 \%$ of patients with familial hemophagocytic lymphohistiocytosis (FHL), cytotoxic granules are defective in their functional perforin content (FHL2); in another 30\% of the patients, cytotoxic granules are defective in their priming state and thus secretion (FHL3). Defective RAB27A in patients with Griscelli syndrome 2 impairs terminal transport and thus exocytosis of the lytic granule contents. X-linked lymphoproliferation and polymerization of perforin are represented with a question mark because there is no experimental proof that they act as represented in this scheme.

Cytomegalovirus infection, or viral hepatitis, and the diagnosis is further complicated by the association of these infections with HS. ${ }^{25,26}$ Biologic alterations include cytopenia, especially anemia and thrombocytopenia. Liver dysfunction, hypertriglyceridemia, hyponatremia, hypofibrinogenemia, and elevated ferritin levels can also occur. Uncontrolled proliferation of $\mathrm{T}$ cells exhibiting the activation markers CD25 and human leukocyte antigen (HLA) class II and activation of macrophages that phagocytose 
Table 1

\begin{tabular}{|c|c|}
\hline Clinical features & $\%$ \\
\hline Fever & $80-100$ \\
\hline Splenomegaly & $55-100$ \\
\hline Hepatomegaly & $45-97$ \\
\hline Lymphadenopathies & $17-52$ \\
\hline Rash & $19-65$ \\
\hline $\begin{array}{l}\text { CNS symptoms (seizures, } \\
\text { confusion etc...) }\end{array}$ & $19-47$ \\
\hline Abdominal pain, distention & 50 \\
\hline \multicolumn{2}{|l|}{ Laboratory abnormalities } \\
\hline Anemia & $89-100$ \\
\hline Thrombocytopenia & $82-100$ \\
\hline Neutropenia & $58-87$ \\
\hline Hypertriglyceridemia & $59-100$ \\
\hline Hypofibrinogenemia & $19-85$ \\
\hline Hyperbilirubinemia & 74 \\
\hline DIC and increased d-dimers & $20-65$ \\
\hline Pathology findings & $\%$ \\
\hline $\begin{array}{l}\text { Needle aspirate or biopsy of bone marrow, liver, spleen, } \\
\text { lymph node: }\end{array}$ & $80-90 \%$ \\
\hline $\begin{array}{l}\text { - Organ infiltration by activated T cells mostly of the CD8 lineage } \\
\text { (CD25 and HLA class II expression) and macrophages) } \\
\text { - Hemophagocytosis } \\
\text { - Indication of potential trigger (infection, malignancy...) }\end{array}$ & $\begin{array}{l}\text { Serial aspirate(s)/biopsy(ies) may be } \\
\text { needed to ascertain HS }\end{array}$ \\
\hline \multicolumn{2}{|l|}{ Lumbar puncture: } \\
\hline Pleiocytosis with activated T cells and/or macrophages & $\sim 45 \%$ \\
\hline Hemophagocytosis & $\begin{array}{l}\text { May be positive even in the absence of } \\
\text { clinical CNS involvement }\end{array}$ \\
\hline
\end{tabular}

blood cells are a hallmark of this syndrome. Because of their "homing" to tissues, especially those of the reticuloendothelial system, phenotyping of circulating blood lymphocytes is often inconclusive and should not lead to the exclusion of a diagnosis of HS. A consistent immunologic finding in active phases of HS is impaired cytotoxic activity of NK cells. ${ }^{27,28}$ Activated T cells and macrophages infiltrate multiple organs, and histopathologically, hemophagocytosis is seen in bone marrow, spleen, liver, lymph nodes, and occasionally the CNS and skin. In the brain, the inflammatory cells form perivascular foci, suggesting a blood-derived tissue infiltration. Activated macrophages may engulf (phagocytose) erythrocytes, and leukocytes, as well as platelets, their precursors, and cellular fragments. These cells appear to be "stuffed" with other blood cells. In the presence of strong clinical and biologic suspicion of HS, it is important that pathologic analysis be repeated if results are initially negative. Immune cell infiltration results in massive tissue necrosis, organ failure, and death in the absence of effective treatments.

\section{Etiology}

Based on an inheritance pattern, HS can be divided into inherited (or primary) HS and acquired (or reactive) HS. 


\section{Inherited $\mathrm{HS}$}

Features that suggest inherited HS include occurrence at a young age (mostly before the age of 3 years although late onset has also been observed); positive family history and previously affected family members; parent consanguinity or parents from a highly hereditary geographic region or ethnic community; and defective NK-cell activity, even in remission phases of HS.

\section{Familial Hemophagocytic Lymphohistiocytosis}

Familial hemophagocytic lymphohistiocytosis (FHL) was first described by Farquhar and Claireaux as familial erythrophagocytic lymphohistiocytosis. ${ }^{29}$ The incidence of FHL has been estimated to be 1 in 50,000 births..$^{30,31}$ Overwhelming HS is the distinguishing and isolated feature in this disorder; there are no other associated signs, unlike the other inherited forms. Symptoms of HS are usually evident within the first 3 months of life and can even develop in utero. Rare cases with delayed onset have been observed. HS most often occurs in previously healthy young children, which suggests the need for an exogenous trigger prior to the onset of clinical manifestations. In susceptible children, infection with intracellular pathogens (viral and fungal, among others) is the most likely trigger for disease manifestation. ${ }^{32} \mathrm{HS}$ in FHL is invariably lethal unless treatment with allogeneic stem-cell transplantation is performed. ${ }^{33}$ Previously, linkage analysis using homozygosity mapping in four hereditary FHL families of Pakistani descent identified a locus (FHL1) on chromosome 9q21.3-22. ${ }^{34}$ However, no causative gene has been so far associated with this locus. Association of this locus with FHL seems restricted to Pakistani families although not all FHL cases in Pakistani families segregate with this locus. ${ }^{35}$ Using genome wide linkage analysis, two additional loci have been identified on chromosomes 10q21-22 (FHL2) ${ }^{36}$ and 17q25 (FHL3), ${ }^{35}$ and there is further evidence of additional genetic heterogeneity and of a yet-undefined gene or genes (G. de St Basile, unpublished data).

\section{FHL2: Perforin Deficiency}

The cytolytic effector perforin, present in cytotoxic granules, was the first gene identified as causing
FHL ${ }^{37}$ As a consequence of perforin gene mutations, perforin protein expression is diminished to barely detectable in cytotoxic granules, ${ }^{32,37,38}$ leading to defective cytotoxic activity. In normal cells, following release from lytic granules, perforin is thought to oligomerize in order to form a pore-like structure in the target cell membrane, analogous to the $\mathrm{C} 9$ component of complement. ${ }^{22}$ Failure of perforin activity is etiologically linked to the development of FHL, and its deficiency accounts for one-third of patients with FHL.

\section{FHL3: Munc13-4 Deficiency}

Patients whose disease is associated with $\mathrm{FHL} 3$ locus present typical features of FHL and are indistinguishable from patients with a perforin (ie, FHL2) defect. In patients with FHL3, however, perforin is normally expressed and is functional. FHL3 was found to be associated with mutations in the gene $U n C 13 D$ encoding for hMunc13-4, a member of the Munc13-UNC13 family. ${ }^{35}$ Six different hMunc13-4 mutations have so far been identified in patients with FHL3 from seven different families. Studies of the exocytosis of cytotoxic granules in lymphocytes from patients with FHL3 mutations showed that Munc13-4 is required for the release of the lytic granule contents but not for other secretory pathways, including the secretion of IFN- $\gamma$ from $T$ cell antigen receptor (TCR)-activated lymphocytes..$^{35}$ Thus, hMuncl3-4 is an essential effector of the cytolytic granule pathway. Munc13-4-deficient lymphocytes can make normal contacts with target cells, stable conjugates, and polarize the lytic machinery as effectively as do control lymphocytes. However, when Munc 13-4 is lost in CTLs, cytotoxic granules dock at the membrane in the immunologic synapse but are not released (see Figure 2B). This supports a role for Munc 13-4 at a late step of this pathway in exocytosis subsequent to docking. Munc13-4 is most probably required at a priming step of lytic granule secretion, following granule docking and preceding plasma granule membrane fusion. ${ }^{24,39,40}$ Of interest, Munc13-4 is expressed in numerous cell type, including platelets and lungs; however, the phenotype of patients with FHL3 is not different from that of patients with perforin deficiency. 


\section{Other Molecular Defects}

Underlying FHL

Perforin and Munc 13-4 deficiencies account for only two-thirds of patients with FHL. Other genes are certainly involved and need further investigation. Analyses of new hereditary families with affected siblings that harbour no perforin or Munc13-4 mutations are needed and should outline other genes that are responsible for FHL.

\section{Chédiak-Higashi Syndrome}

A Cuban pediatrician first described ChédiakHigashi syndrome in $1943 .{ }^{41}$ Hematologic abnormalities associated with this rare disorder were subsequently reported in 1952 by Chediak, ${ }^{42,43}$ and the presence of monstrous cytotoxic granules was emphasized by Higashi in $1953 .{ }^{44}$ Chédiak-Higashi syndrome is a rare autosomal recessive disorder (approximately 200 cases are reported in the world) characterized by variable degrees of occulocutaneous albinism, easy bruising and bleeding as a result of deficient platelet dense bodies, recurrent infections with neutropenia and impaired neutrophil functions (including impaired chemotaxis and bactericidal activity), and abnormal NK-cell function. ${ }^{44}$ Neurologic involvement is variable but often includes peripheral neuropathy, and patients with a milder expression of the disease are frequently referred for this symptom in adulthood. Most patients are diagnosed during the first decade of life. Death often occurs in the first decade of life from infection, bleeding, or development of HS. HS is often triggered by ongoing intracellular infection, including infection with herpesviruses. The hallmark of Chédiak-Higashi syndrome is the presence of huge cytoplasmic granules in circulating granulocytes and many other cell types (Figure 4A; see also Figure 2B). These granules are peroxidase positive and contain lysosomal enzymes, suggesting that they are giant lysosomes or (in the case of melanocytes) giant melanosomes. The underlying defect in Chédiak-Higashi syndrome remains elusive, but the disorder can be considered as a model for defects in vesicle formation, fusion, or trafficking. The normal degradative functions of this compartment appear to be intact. The defect is apparent only in cells that require secretion of their

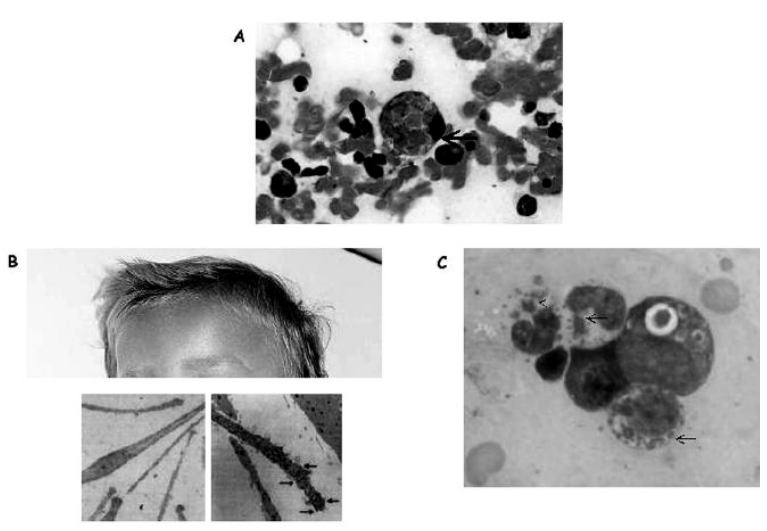

Figure 4 Illustration of hemophagocytosis and the most prominent extrahematologic features of Griscelli and Chédiak-Higashi syndromes. A, Hemophagocytosis in the bone marrow of a patient with familial hemophagocytic lymphohistiocytosis; arrow indicates an activated macrophage that has ingested several red blood cells. $B$, Partial view of the head of a child with Griscelli syndrome 2, shown to emphasize the ashengrey colour of hair. Electron microscopy images of a normal hair (left panel) and a hair of a person with Griscelli syndrome (right panel) are shown below; arrows indicate clumps of melanin specific for this disease. A defect in any of the proteins (myosin Va, RAB27A, or melanophilin) leads to identical pigmentary dilution in the three forms of Griscelli syndrome and their mouse models. $C$, Blood smear taken from a patient with Chédiak-Higashi syndrome. Arrows indicate large granules present in all cell lineages that orient the diagnosis toward Chédiak-Higashi syndrome.

lysosomes. This is seen in melanosomes, major histocompatibility complex class II compartments, azurophilic granules, and lytic granules, yet no dysfunction is seen in conventional secretory cells that use secretory granules. This is consistent with a crucial role for the Chediak protein in cells that have cytotoxic granules. The protein defective in Chédiak-Higashi syndrome patients and in the beige mouse model has been identified as the 419 kD Chédiak-Higashi syndrome 1/LYST protein. ${ }^{45,46}$ Given the length $(13.5 \mathrm{~kb})$ of the Chédiak-Higashi syndrome 1 gene ( $\mathrm{CHS} 1)$, mutation screening is a difficult task. In patients with the classic form of Chédiak-Higashi syndrome, nonsense or frameshift mutations leading to early truncation of the protein have been reported. In contrast, missense mutations were identified in the few patients 
with a milder clinical course. ${ }^{45-48}$ The exact role of LYST is still unknown. Overexpression of LYST in deficient fibroblasts induces the production of unusually small lysosomes, suggesting that LYST is involved in lysosome fission. Recently, the domain of LYST that controls lysosome size has been mapped. ${ }^{49}$ The seemingly contradictory roles of increased membrane fusion (or decreased membrane fission), leading to enlarged lysosomes, and the inability of lysosomes to fuse at the plasma membrane during secretion can be explained if LYST acts to regulate membrane fusion/fission events. This is compatible with recent findings that LYST interacts with a soluble N-ethylmaleimide-sensitive factor attachment protein receptor (SNARE protein) involved in membrane fusion..$^{50}$ At what step of the exocytic pathway does the function of Chédiak-Higashi syndrome/LYST in membrane fusion/fission events operate remains to be determined and is the object of current work by different groups. Allogeneic stem cell transplantation remains the only cure for children with Chédiak-Higashi syndrome. Engraftment of donor cells ensures the correction of hematologic abnormalities. However, CNS signs associated with Chédiak-Higashi syndrome are not treated through this procedure and increase with the patient's age. In a recent report, 14 patients with Chédiak-Higashi syndrome who underwent successful stem cell transplantation early in the course of their disease showed progressive neurologic dysfunction with neurologic deficits or low cognitive abilities. These neurologic problems are not linked to transplant-related morbidity or previous infections; they are caused by the underlying molecular defect and indicate that the benefits of correcting the hematologic and immunologic aspects of the disease must be weighed against the limitation of neurologic and cognitive deficits occurring later in life despite successful transplantation. ${ }^{51}$

\section{Griscelli Syndrome}

First described in 1978 as a syndrome associating immunodeficiency with partial albinism, Griscelli syndrome is an autosomal recessive heterogeneous disorder characterized by a pigmentary dilution, a silvery gray sheen of the hair, and a typical pattern of uneven distribution of large pigment granules that is easily detectable by light-microscopic examination ${ }^{52,53}$ (see Figure 4B). Sunexposed areas of the patients' skin are often hyperpigmented, and microscopic analysis of the dermoepidermal junction will detect an accumulation of mature melanosomes in melanocytes, contrasting with the hypopigmented surrounding keratinocytes..$^{52}$ Although this is a rare disease, three genetic forms of the syndrome have been defined, as follows:

1. Griscelli syndrome 1 (mutations in MYO5A, a gene present on $15 \mathrm{q} 21$ ): pigmentary abnormalities associated with neurologic features, including hypotonia and developmental delay. ${ }^{54}$

2. Griscelli syndrome 2 (mutations in $R A B 27 A$, a gene adjacent to $M Y O 5 A$ on $15 \mathrm{q} 21)^{55}$ : the only form associated with HS and the only one to be further discussed in this review.

3. Griscelli syndrome 3 (mutations in melanophilin): isolated pigmentary abnormalities. ${ }^{56}$

RAB27A plays an important role in melanocytes and in cytotoxic function. Like patients with Chédiak-Higashi syndrome, patients with Griscelli syndrome 2 exhibit marked hypopigmentation, but unlike Chédiak-Higashi syndrome patients, their lysosomes are normal in size. In CTLs and melanocytes, RAB27A is required at a late stage of secretion in order to leave the microtubule cytoskeleton and dock at the plasma membrane. ${ }^{13,24}$ However, the precise function of RAB27A differs in melanocytes and CTLs. In melanocytes, RAB27A associates with the melanosomal membrane and recruits melanophilin, a synaptotagmin-like protein, which in turn interacts with myosin $\mathrm{Va}$, an unconventional myosin motor that moves along the actin cytoskeleton and tethers the melanosome at the plasma membrane ready for pigment delivery. In CTLs, RAB27A does not interact with either melanophilin or myosin Va, and CTLs with mutated myosin Va or melanophilin do not have impaired cytotoxic activity. CTLs lacking RAB27A contain cytotoxic granules of normal size and morphology that appear to polarize toward the MTOC nor- 
mally (see Figure 4B). However, electron microscopy reveals that these granules remain aligned, one behind the other, along the microtubules leading to the MTOC. They are unable to dock at the plasma membrane in RAB27A-deficient CTLs, and together these observations suggest that RAB27A is required for the granules to detach from microtubules before they can dock at the plasma membrane. One important lesson to emerge from studies of both the Chédiak-Higashi and Griscelli syndromes is that although key proteins such as RAB27A play roles in lysosomal secretion in many cell types, the precise composition of the secretory machinery varies from one cell to another.

\section{X-Linked Lymphoproliferative Syndrome}

$\mathrm{X}$-linked lymphoproliferative syndrome (also called Purtilo's disease) was first characterized by an extreme susceptibility to EBV infection..$^{57}$ Patients with this syndrome present with three main phenotypes: fatal infectious mononucleosis, malignant B-cell lymphomas, and dysgammaglobulinemia. A patient can develop more than one phenotype, particularly after exposure to EBV. More than $70 \%$ of patients with X-linked lymphoproliferative syndrome die before the age of 10 years, and all patients with this disease die by the age of 40 years. HS in these patients is fulminant and seems to be exquisitely triggered by the encounter of patients with EBV. X-linked lymphoproliferative syndrome can result from mutations in the small SH2-domain-containing protein, SAP/SH2D1A/DSHP, which can associate with several cell surface receptors of the SLAM family of immune receptors. Recent findings indicate that SAP participates in intracellular signalling in immune cells and is required for the function of SLAM as a consequence of its capacity to promote the recruitment and activation of the Srcrelated protein tyrosine kinase FynT ${ }^{58}$ Of interesting, several studies have identified a role of SAP in NK cell-mediated cytotoxicity through its association with members of the SLAM family (ie, 2B4 and NTB-A, which are both expressed on NK cells and some CD8+ T cells). ${ }^{59,60}$ Several studies show that engagement of 2B 4 or NTB-A on these cells activates degranulation-mediated cytotoxic- ity. ${ }^{61}$ In contrast, when SAP is absent, these receptors play an inhibitory role in cytotoxicity ${ }^{62}$ Thus, cells from patients with X-linked lymphoproliferative syndrome exhibit a severe cytotoxic defect through the engagement of these receptors, ${ }^{62-64}$ which could compromise their ability to kill EBVinfected B cells and could favour the occurrence of HS.

\section{Steps in Diagnosing Primary HS}

Distinguishing primary forms from secondary forms of HS is important not only in terms of genetic counselling for this condition but also for determining the appropriate therapeutic intervention. The occurrence of HS at a young age should instigate the search for a genetic cause. Microscopic analysis of the hair shaft is an easy and reliable test for diagnosing Griscelli syndrome and Chédiak-Higashi syndrome. In both conditions, pigmentation dilution is characteristic, but there is larger clumping of pigment in the hair shafts of a patient with Griscelli syndrome than in the hair shafts of a patient with Chédiak-Higashi syndrome (see Figure 4B). Carriers of these syndromes have normal pigmentation. The presence of giant intracytoplasmic granules in all cells from the hematopoietic lineage is a hallmark of ChédiakHigashi syndrome; this is easy to identify in a blood smear (see Figure 4C) and rapidly ensures diagnosis. If pigmentation dilution orients toward Griscelli syndrome, sequencing of the $R A B 27 A$ gene allows confirmation of that diagnosis. In the absence of HS, molecular diagnosis of Griscelli syndrome is important for ruling out potential RAB27A deficiencies, which should be treated by allogeneic stem cell transplantation. In ChédiakHigashi syndrome, given the length of the CHS1 gene, mutation screening is not used as a routine test for diagnosis and genetic counselling. An unambiguous diagnosis of this condition can be made without need for further genetic testing, based on the characteristic hypopigmentation of hair shafts and the presence of intracellular giant granules. However, for genetic counselling of families, segregation analysis of polymorphic markers linked to the Chédiak-Higashi syndrome locus on chromosome 1q43.2 in the family can be used. In nonconsanguineous families, this approach 
requires the availability of a sample of deoxyribonucleic acid (DNA) from both parents and from the patient to determine the affected haplotype in the family. When parents are related, the identification of a shared haplotype at the ChédiakHigashi syndrome locus in the parents may overcome the unavailability of a DNA sample from the patient. When HS is not associated with hypopigmentation, the biggest difficulty lies in differentiating between the primary (inherited) disease (FHL) and a secondary HS disease. A positive family history with previously affected family members and/or consanguinity of the parents is highly suggestive of an inherited form. The availability of biologic samples from family members such as parents and siblings greatly helps the molecular diagnosis of genetic causes by rapid determination of the polymorphic markers segregating with the disease locus. However, the lack of family history is not a reliable criterion for excluding FHL. The study of the cytotoxic activity of T lymphocytes $^{37,40}$ is a reliable test with which to diagnose the genetic forms of HS. About $30 \%$ of FHL cases result from a perforin defect, which can be rapidly identified by immunofluorescence analysis of perforin expression in resting cytotoxic cells. In fact, the great majority of mutations so far identified in FHL2 dramatically affect perforin detection. Sequencing of the perforin gene will confirm the diagnosis of FHL. Another group of FHL cases (about $60 \%$ ) is characterized by defective T-cell cytotoxic activity but normal perforin expression. In half of these cases, sequencing of the MUNC13.4 gene allows identification of FHL from Munc13-4 deficiency. In the rest, the genetic cause is not yet characterized. Defective Tlymphocyte cytotoxic activity is the signature of a primary genetic cause of HS in $90 \%$ of cases. In approximately $10 \%$ of FHL cases, however, defects in T-cell cytotoxic activity cannot be evidenced. In the absence of family history, these forms cannot be clearly distinguished from secondary forms of HS, and they remain a diagnostic challenge.

Finally, the diagnosis of X-linked lymphoproliferative syndrome should be confirmed by sequencing of the $S A P$ gene and potentially by the analysis of SAP protein expression, with the knowledge that a significant number of patients with the X-linked lymphoproliferative syndrome-like phenotype do not have mutations in this gene but potentially do have mutations in other yet-uncharacterized genes..$^{59,60}$

\section{Acquired HS}

Acquired HS can be as clinically, biologically, and pathologically overwhelming as can inherited HS. In remission phases of HS, patients with acquired HS have normal NK-cell activity.

\section{Rheumatoid Diseases}

In the early 1980 s, several reports described patients with systemic-onset juvenile rheumatoid arthritis (JRA) in whom a severe coagulopathy resembling disseminated intravascular coagulation developed. ${ }^{65}$ Such a coagulopathy was often associated with changes of mental status, hepatosplenomegaly, increased serum levels of liver enzymes, and sharp falls in blood counts and erythrocyte sedimentation rates. In 1985, Hadchouel and colleagues linked these symptoms to massive proliferation of activated nonneoplastic macrophagic histiocytes with prominent hemophagocytic activity. ${ }^{66}$ The term macrophage activation syndrome (MAS) was eventually introduced in 1993 by Stephan and colleagues in a follow-up report originating from the same centre. ${ }^{5}$ Over the following years, several more reports from various countries described a number of patients with very similar symptoms. MAS, reactive hemophagocytic lymphohistiocytosis (HLH), and HS are different denominations of the same clinical entity. Although HS has also been observed in a small number of patients with polyarticular JRA and in those with collagen diseases (including lupus, vasculitis, Kawasaki disease, dermatomyositis, and panniculitis), it is most commonly seen in patients with the systemic form of JRA. ${ }^{67-69}$

It is still unclear why some individuals with these rheumatologic disorders develop MAS during the course of their disease. A pathogen trigger is often present, initiating HS in this setting. In a study including seven patients with MAS, decreased NK-cell activity was observed in all patients, and decreased perforin expression was found in two of the seven patients despite a nor- 
mal perforin 1 gene sequence..$^{70}$ Decreased expression of SAP transcript has also been reported in peripheral T cells of patients with JRA. ${ }^{71}$ A transient inhibition of the cytotoxic granule pathway may be sufficient in a setting of immune system disturbances such as systemic JRA or other rheumatoid disorders. In these patients, pathogens may not be properly cleared during the first wave of infection, thus promoting the development of very potent immune system activation and HS. The hypothesis that impaired cytotoxic functions and lack of immunoregulation by NK cells result in MAS/HS in rheumatoid disorders remains to be proven. What is clear is that both inherited HS and acquired HS share the same immunologic abnormalities in terms of macrophage and T-lymphocyte activation and expansion and should initially be treated similarly, as overwhelming lymphocyte activation.

\section{Infection-Associated HS}

In 1979, HS was described in a cohort of patients who had serologic evidence of recent viral infections, and virus-associated HS was proposed as a distinct clinical entity. ${ }^{72}$ Subsequently, HS has been reported in association with a variety of infections, and the term "reactive hemophagocytic syndrome" has been suggested to distinguish HS associated with an identifiable infectious or noninfectious cause from its hereditary forms. However, the reactive and hereditary forms of the disease are difficult to distinguish; for example, patients with familial forms of HS may have HS after a documented infection. Case reports and case series on the association of infections and HLH have been summarized. ${ }^{26}$ The most frequent pathologic conditions that can cause HS are infection by viruses (including viruses of the herpes family, human immunodeficiency virus, and adenovirus), fungal infections, bacterial and mycobacterial infections, and parasitic infection (including visceral leishmaniasis and toxoplasmosis). ${ }^{11,73-86}$ Special emphasis should be given to EBVassociated HS. With the exclusion of inherited disorders, this form of acquired HS seems more severe than other types of infection-associated HS. It is a recurrent overwhelming HS, and its outcome is often fatal despite optimal management.
The etiology of this syndrome remains poorly understood (see below). The resolution of HS following treatment of the infection suggests that in many cases, HS is secondary to the underlying infection. A diagnosis that takes into account all of the underlying diseases associated with HS would be impractical, and formal guidelines for evaluating patients with suspected infectionassociated HLH have not been established. Extensive testing for underlying infecting organisms should be guided by epidemiologic data and the patient's medical history.

\section{Malignant Disorders and HS}

HS has been shown to be associated with malignant histiocytosis. ${ }^{72}$ In regard to malignancies, HS is mostly encountered in patients with T-cell malignancies, especially T-cell lymphomas. ${ }^{87-89}$ It can be present at onset or during the course of treatment and usually implies relapse or escape of the leukemic clone from chemotherapeutic agents. ${ }^{90}$ Although $\mathrm{T}$ lymphocytes lack the putative EBV receptor CD21, the presence of episomal EBV genome in T-cell lymphomas ${ }^{25,91-93}$ and T lymphocytes from patients with virus-associated HS is has been well described.$^{94} \mathrm{EBV}$-positive $\mathrm{T}$-cell lymphomas appear to elaborate TNF- $\alpha$ more frequently than either EBV-positive B-cell lymphomas or EBV-negative T-cell lymphomas. ${ }^{25,93}$ Lay and colleagues induced the expression of CD21 in T-lymphoma cell lines and subsequently infected these cells with EBV. High levels of TNF- $\alpha$, IFN- $\gamma$, and IL- $1 \alpha$ were secreted by these cells after EBV infection; when the lymphocytes were cocultured with monocytes, enhanced phagocytosis by monocytes was observed. The enhanced phagocytosis was eliminated by the addition of antibodies against TNF- $\alpha$ and IFN- $\gamma \cdot{ }^{25,93}$ Clonal expansion of EBV-infected $\mathrm{T}$ lymphocytes has been demonstrated in both EBV-associated HS and EBV-positive T-cell lymphoma by the presence of homogeneous viral terminal repetitive sequences. EBV-infected cells stain positive for such T-lymphocyte markers as CD45RO and TCR- $\beta$. Clonality of infected T lymphocytes is further suggested by the finding of monoclonal re-arrangements of the TCR- $\beta$ gene in EBV-associated HS. ${ }^{95}$ The distinction between 
the monoclonal proliferation of $\mathrm{T}$ lymphocytes seen in EBV-associated HS and EBV-positive T-cell lymphomas may describe the extremes of a spectrum of disordered T-lymphocyte proliferation and cytokine elaboration following EBV infection of T lymphocytes. It is unclear whether clonal proliferation of Tlymphocytes occurs in HS that is associated with pathogens other than EBV. The fact that these syndromes seem more likely to resolve with control of the underlying infection suggests that this may not be the case. ${ }^{72}$

\section{Treatment of HS}

HS is a severe disease that is associated with considerable morbidity and mortality unless proper management is undertaken. Early recognition of this syndrome and immediate aggressive therapeutic intervention are critical and may prevent the development of the full-blown syndrome. Immunosuppression-based therapeutic strategies have revolutionized management and clearly outline the central role of $\mathrm{T}$ cells in disease initiation and maintenance.

\section{Primary HS}

Treatment of primary HS needs to be considered in two steps: (1) managing current HS and potential infectious triggers and (2) preventing HS from recurring. There are currently two major therapeutic options for the management of HS in hereditary disorders. A historical line of treatment with guidelines developed by the Histiocyte Society and summarized in the HLH-94 treatment protocol is available..$^{96}$ On the basis of the former suspicion that HS might be caused by malignant histiocytes, this group previously instated a regimen associating high-dose parenteral corticosteroids and etoposide injections. More recently, Cyclosporin A was added to this regimen, along with other modifications. Etoposide is a chemotherapeutic agent that kills histiocytes (tissue macrophages) and was chosen accordingly. However, this drug has been associated with secondary leukemias and liver toxicity, as well as a dose-dependent increase in the rate of veno-occlusive disorders following stem cell transplantation in patients receiving this medication. Although it is effective in treating HS, this protocol does not fully take into consideration the key role of T-cell activation in HS. Moreover, to our group, the use of etoposide seems unjustified as a first line of action because of its potential side effects and its lack of a specific target, and thus we feel that its use should be restricted to HS associated with malignancies. Data from molecular analyses of the known causes of hereditary disorders associated with HS undoubtedly show that HS is an immunologic disease and outline $\mathrm{T}$ cells as key targets for therapeutic intervention. A more recent protocol that takes this aspect into consideration offers an immunosuppressive regimen combining high-dose antithymocyte globulins (ATGs), high-dose parenteral methylprednisolone, and cyclosporin A.,33 (Thymocytes are lymphocytes; ATG derived from rabbits is preferred to that derived from horses.) This approach has proved repeatedly effective in obtaining good-quality remissions of HS and is currently part of the guidelines of the European Society of Immunodeficiencies, part of the European Group for Bone and Marrow Transplantation (EBMT). ${ }^{33}$ Special attention should be given to CNS involvement in HS cases. Beside high-dose corticosteroids, the medications used have poor CNS penetration. In the case of a positive lumbar puncture (LP), repeated local injections of methotrexate in doses similar to those administered for childhood acute lymphoblastic leukemia are needed. Even if the LP is negative, CNS prophylaxis should be instituted until the time of stem cell transplantation (Table 2).

The only therapeutic means of preventing HS from reoccurring is hematopoietic stem cell transplantation (HSCT). From the moment the diagnosis of HS is established and an inherited cause is suspected, a search for the most appropriate source of hematopoietic stem cells should be undertaken, even if molecular investigations to characterize the defect are ongoing. A diagnostic and therapeutic dilemma remains for sporadic de novo (nonfamilial) cases of FHL that do not involve FHL2 or FHL3 as a molecular defect in children who are over 3 years of age and exhibit borderline NK-cell 
Table 2

Guidelines of the European Bone Marrow Transplant/European Society for Inborn Errors group for the treatment of inherited hemophagocytic syndromes.

1- Intra-venous Methylprednisolone:

- day 1 to day 2 (48 hours) $5 \mathrm{mg} / \mathrm{kg} / \mathrm{d}$ divided in 2 doses

- day 3 to day 4 (48 hours) $3 \mathrm{mg} / \mathrm{kg} / \mathrm{d}$ divided in 2 doses

- day 4 2mg/kg /d till disease control and serum levels of ciclosporine A of 200-300 ng/ml, then taper to full stop previous to stem cell transplantation.

2- Rabbit anti-thymocyte globulins (ATG)

- Day1 to day 5 (5 days) $10 \mathrm{mg} / \mathrm{kg} / \mathrm{d}$ IV infusion over 6-12 hours

3- Cyclosporine A

- begin 48-72 hours after initiating ATG

- $3 \mathrm{mg} / \mathrm{kg} / \mathrm{d}$ in continuous or in 1 hour BID infusion

- aim is to have serum levels within $200-300 \mathrm{ng} / \mathrm{ml}$

- give oral treatment whenever disease is controlled and maintain until the beginning of the conditioning regimen for stem cell transplantation.

4- Intrathecal Methotrexate (MTX)

Doses: as per acute lymphoblastic leukemia protocols,

$\begin{array}{ll}6 \mathrm{mg} & \text { age } 0-1 \text { year } \\ 8 \mathrm{mg} & \text { age } 1-2 \text { years } \\ 10 \mathrm{mg} & \text { age } 2-3 \text { years } \\ 12 \mathrm{mg} & \text { age }>3 \text { years }\end{array}$

Regimen: DO NOT EXCEED 8 IT treatments

Presence of Central Nervous system involvement on imaging or lumbar tap

2 LPs/week for 2 weeks

$1 \mathrm{LP} /$ week optimally up to Stem Cell Transplant

Absence of Central Nervous Involvement at diagnosis

$1 \mathrm{LP} /$ week optimally up to Stem Cell Transplant

activity. In all other cases, HSCT is mandated and will exclusively correct hematologic abnormalities. Ideally, an HLA genotypically identical nonaffected sibling is available, and HSCT with this donor type is successful in $85 \%$ of cases. . $^{33,97,98}$ Matched unrelated T-depleted haploidentical or cord blood transplantations have also been performed with variable success and a 5-year survival rate ranging from 55 to $75 \%$, depending on the series. ${ }^{33,97,98}$ In $20 \%$ of cases, two iterative HSCTs were required to ensure engraftment or maintain chimerism. ${ }^{33}$ We here also support a conditioning regimen that does not include etoposide and combines rabbit ATG with conventional busulfan/cyclophosphamide. Tables 2 and 3 list guidelines recommended by the EBMT Inborn
Errors Working Party for the treatment of HS as well as for the conditioning of patients with FHL, Griscelli syndrome 2, and Chédiak-Higashi syndrome.

\section{Acquired HS}

\section{Rheumatoid Disorders}

The treatment strategy for MAS/HS is based on the parenteral administration of high doses of methylprednisolone.$^{67}$ After normalization of coagulation and hematologic abnormalities, a slow taper is then performed. Frequently, MAS is resistant to corticosteroids, and cases with fatal evolutions despite the administration of massive 
Table 3

Guidelines of the European Bone Marrow Transplant/European Society for Inborn Errors group for

conditioning regimen for hematopoietic stem cell transplant of inherited hemophagocytic syndromes.

In all cases it is strongly recommended to obtain optimal control of hemophagocytic syndrome previous to undertaking hematopoietic stem cell transplant.

A- Genotypical identical donor

- Rabbit ATG day-14 to -10 (5 days), dose $10 \mathrm{mg} / \mathrm{kg} / \mathrm{day}$

- Busulfan: day -10 to -7 ; age $<6 y 5 \mathrm{mg} / \mathrm{kg} / \mathrm{day} X$ X;age $>6 \mathrm{y} 4 \mathrm{mg} / \mathrm{kg} /$ dayX 4

- Cyclophosphamide: day -5 to $-2 ; 50 \mathrm{mg} / \mathrm{kg} / \mathrm{day} \mathrm{X} 4$

B- Matched unrelated donor/Phenotype identical related donor/HLA-nonidentical haplo or mismatched family donor.

- Rabbit ATG day-14 to -10 (5 days), dose $10 \mathrm{mg} / \mathrm{kg}$

- Busulfan: day -10 to -7 ; age $<6 y 5 \mathrm{mg} / \mathrm{kg} /$ day X 4;age $>6 y$ 4mg/kg/dayX 4

- Cyclophosphamide: day -5 to $-2 ; 50 \mathrm{mg} / \mathrm{kg} /$ day X 4

- Positive selection of CD34+ cells in Stem cell source aiming to have T cell counts $<10^{4} / \mathrm{kg}$

- Ciclosporine A if T cell counts above 104/kg despite positive selection of CD34+ cells

doses of steroids have been described.$^{67}$ Parenteral administration of cyclosporin $\mathrm{A}$ at a dosage of 2 to $8 \mathrm{mg} / \mathrm{kg} / \mathrm{d}$ has revolutionized outcomes. . $^{4,87,99}$ There are anecdotal reports on the use of etoposide and intravenous immunoglobulins for treatment of MAS; the results conflict, and we do not suggest the use of these drugs in MAS cases. Therapies that inhibit T-cell function may be expected to make patients with JRA more susceptible to the development of HS in the face of infection; on theoretical grounds, therefore, one may recommend that such drugs be reduced transiently during episodes of infection.

\section{Infection}

A short course of intravenous methylprednisolone $(1-2 \mathrm{mg} / \mathrm{kg} / \mathrm{d})$ is often sufficient in treating HS associated with bacterial, parasitic, and fungal infections. This treatment is given in concert with adequate antiinfectious agents, and it must be administered for the shortest time necessary for correction of the most life-threatening symptoms associated with HS because of the added immunosuppression in the context of severe infection. EBV-associated HS remains a therapeutic challenge and often has a fatal outcome despite aggressive management. We suggest that this form be treated as HS associated with inherited disorders. New antiviral drugs such as cidofovir or ribavirin may be useful; however, the results achieved with these drugs in the treatment of other B lymphoproliferative disorders associated with EBV are conflicting. Consideration should be given to the use of IVIG in infection-associated HS, to minimize the risk of immunosuppression.

\section{Malignancies}

In malignancies, a short pulse of intravenous steroids $(2 \mathrm{mg} / \mathrm{kg} / \mathrm{d})$ is usually administered along with conventional chemotherapy adapted to the underlying disease. With the destruction of the malignant clone, resolution of HS is often seen and no additional treatment is needed. Etoposide $\left(100 \mathrm{mg} / \mathrm{m}^{2}\right.$ per dose) as per the HLH-94 protocol ${ }^{96}$ can be considered in case of resistance to corticosteroids.

\section{Supportive Care}

All HS patients should be hospitalized in tertiary care centers with an intensive care unit at hand. Inherited disorders often have overwhelming rapidly progressive HS; however, this can also be encountered in cases of acquired HS. Priority should be given to obtaining central venous line access, instating transfusional support, and managing (whenever possible) infectious triggers. Managing hyponatremia and kidney and liver fail- 
ures should be ad hoc, especially because of the unavoidable use of nephrotoxic antiinfectious and immunosuppressive drugs.

\section{Conclusions}

Because so many immunologic, neoplastic, genetic, and infectious disorders may be associated with hemophagocytic syndrome, the management of this syndrome clearly calls for a multidisciplinary approach among experienced clinicians, pathologists, and microbiologists to define the diagnosis and the precipitating or underlying illnesses. Another big challenge is to characterize, on a molecular level, yet-unknown genes responsible for diseases such as familial hemophagocytic lymphohistiocytosis and X-linked lymphoproliferative syndrome. This should also improve the understanding of the fine regulation of T-cell responses, an area of major therapeutic impact.

\section{Acknowledgement}

The authors wish to acknowledge Dr. Alain Fischer, who initiated and furthered a substantial number of these studies.

\section{References}

1. Scott R, Robb-Smith A. Histiocytic medullary reticulosis. Lancet 1939;2:194-8.

2. Henter JI, Elinder G, Ost A. Diagnostic guidelines for hemophagocytic lymphohistiocytosis. The FHL Study Group of the Histiocyte Society. Semin Oncol 1991;18:29-33.

3. Henter JI, Elinder G. Familial hemophagocytic lymphohistiocytosis. Clinical review based on the findings in seven children. Acta Paediatr Scand 1991;80:269-77.

4. Mouy R, Stephan JL, Pillet P, et al. Efficacy of cyclosporine $\mathrm{A}$ in the treatment of macrophage activation syndrome in juvenile arthritis: report of five cases. J Pediatr 1996;129:750-4.

5. Stephan JL, Zeller J, Hubert P, et al. Macrophage activation syndrome and rheumatic disease in childhood: a report of four new cases. Clin Exp Rheumatol 1993;11:451-6.

6. Wong KF, Hui PK, Chan JK, et al. The acute lupus hemophagocytic syndrome. Ann Intern Med 1991;114:387-90.

7. Kadin ME, Kamoun M, Lamberg J. Erythrophagocytic T gamma lymphoma: a clinicopathologic entity resembling malignant histiocytosis. N Engl J Med 1981;304:648-53.

8. Henter JI, Ehrnst A, Andersson J, Elinder G. Familial hemophagocytic lymphohistiocytosis and viral infections. Acta Paediatr 1993;82:369-72.

9. Browett PJ, Varcoe AR, Fraser AG, Ellis-Pegler RB. Disseminated tuberculosis complicated by the hemophagocytic syndrome. Aust N Z J Med 1988;18:79-80.

10. Caksen H, Akbayram S, Oner AF, et al. A case of typhoid fever associated with hemophagocytic syndrome. J Emerg Med 2003;25:321-2.

11. Campo E, Condom E, Miro MJ, et al. Tuberculosis-associated hemophagocytic syndrome. A systemic process. Cancer 1986;58:2640-5.

12. Matzner Y, Behar A, Beeri E, et al. Systemic leishmaniasis mimicking malignant histiocytosis. Cancer 1979;43:398-402.

13. de Saint Basile G, Fischer A. Defective cytotoxic granule-mediated cell death pathway impairs T lymphocyte homeostasis. Curr Opin Rheumatol 2003;15:436-45.

14. Van Parijs L, Abbas AK. Homeostasis and selftolerance in the immune system: turning lymphocytes off. Science 1998;280:243-8.

15. Zinkernagel RM. Localization dose and time of antigens determine immune reactivity. Semin Immunol 2000;12:163-71, 257-344.

16. Haddad E, Sulis ML, Jabado N, et al. Frequency and severity of central nervous system lesions in hemophagocytic lymphohistiocytosis. Blood 1997;89:794-800.

17. Henter JI, Elinder G. Cerebromeningeal haemophagocytic lymphohistiocytosis. Lancet 1992;339:104-7.

18. Henter JI, Nennesmo I. Neuropathologic findings and neurologic symptoms in twenty-three children with hemophagocytic lymphohistiocytosis. J Pediatr 1997;130:358-65.

19. Henter JI, Elinder G, Soder O, et al. Hypercytokinemia in familial hemophagocytic lymphohistiocytosis. Blood 1991;78:2918-22. 
20. Takada H, Ohga S, Mizuno Y, et al. Oversecretion of IL-18 in haemophagocytic lymphohistiocytosis: a novel marker of disease activity. $\mathrm{Br}$ J Haematol 1999;106:182-9.

21. Hasegawa D, Kojima S, Tatsumi E, et al. Elevation of the serum Fas ligand in patients with hemophagocytic syndrome and Diamond-Blackfan anemia. Blood 1998;91:2793-9.

22. Stepp SE, Mathew PA, Bennett M, et al. Perforin: more than just an effector molecule. Immunol Today 2000;21:254-6.

23. de Saint Basile G, Fischer A. The role of cytotoxicity in lymphocyte homeostasis. Curr Opin Immunol 2001;13:549-54.

24. Stinchcombe J, Bossi G, Griffiths GM. Linking albinism and immunity: the secrets of secretory lysosomes. Science 2004;305:55-9.

25. Lay JD, Tsao CJ, Chen JY, et al. Upregulation of tumor necrosis factor-alpha gene by EpsteinBarr virus and activation of macrophages in Epstein-Barr virus-infected T cells in the pathogenesis of hemophagocytic syndrome. J Clin Invest 1997;100:1969-79.

26. Fisman DN. Hemophagocytic syndromes and infection. Emerg Infect Dis 2000;6:601-8.

27. Schneider EM, Lorenz I, Muller-Rosenberger $\mathrm{M}$, et al. Hemophagocytic lymphohistiocytosis is associated with deficiencies of cellular cytolysis but normal expression of transcripts relevant to killer-cell-induced apoptosis. Blood 2002;100:2891-8.

28. Egeler RM, Shapiro R, Loechelt B, Filipovich A. Characteristic immune abnormalities in hemophagocytic lymphohistiocytosis. J Pediatr Hematol Oncol 1996;18:340-5.

29. Farquhar J, Claireaux A. Familial haemophagocytic reticulosis. Arch Dis Child 1952;27:519-25.

30. Henter JI, Elinder G, Soder O, Ost A. Incidence in Sweden and clinical features of familial hemophagocytic lymphohistiocytosis. Diagnostic guidelines for hemophagocytic lymphohistiocytosis. The FHL Study Group of the Histiocyte Society. Acta Paediatr Scand 1991;80:428-35.

31. Dufourcq-Lagelouse R, Pastural E, Barrat FJ, et al. Genetic basis of hemophagocytic lymphohistiocytosis syndrome. Int J Mol Med 1999;4:127-33.

32. Feldmann J, Le Deist F, Ouachee-Chardin M, et al. Functional consequences of perforin gene mutations in 22 patients with familial haemophagocytic lymphohistiocytosis. $\mathrm{Br} \mathbf{J}$ Haematol 2002;117:965-72.

33. Jabado N, de Graeff-Meeder ER, CavazzanaCalvo M, et al. Treatment of familial hemophagocytic lymphohistiocytosis with bone marrow transplantation from HLA genetically nonidentical donors. Blood 1997;90:4743-8.

34. Ohadi M, Lalloz MR, Sham P, et al. Localization of a gene for familial hemophagocytic lymphohistiocytosis at chromosome 9q21.3-22 by homozygosity mapping. Am J Hum Genet 1999;64:165-71.

35. Feldmann J, Callebaut I, Raposo G, et al. Munc13-4 is essential for cytolytic granules fusion and is mutated in a form of familial hemophagocytic lymphohistiocytosis (FHL3). Cell 2003;115:461-73.

36. Dufourcq-Lagelouse R, Jabado N, Le Deist F, et al. Linkage of familial hemophagocytic lymphohistiocytosis to 10q21-22 and evidence for heterogeneity. Am J Hum Genet 1999;64:172-9.

37. Stepp SE, Dufourcq-Lagelouse R, Le Deist F, et al. Perforin gene defects in familial hemophagocytic lymphohistiocytosis. Science 1999;286:1957-9.

38. Kogawa K, Lee SM, Villanueva J, et al. Perforin expression in cytotoxic lymphocytes from patients with hemophagocytic lymphohistiocytosis and their family members. Blood 2002;99:61-6.

39. Feldmann J, Le Deist F, Fischer A, de Saint Basile G. [Munc13-4 is essential for cytolytic granule fusion]. Med Sci (Paris) 2004;20:144-6.

40. Feldmann J, Callebaut I, Raposo G, et al. Munc13-4 is essential for cytolytic granules fusion and is mutated in a form of familial hemophagocytic lymphohistiocytosis (FHL3). Cell 2003;115:461-73.

41. Beguez-Cesar A. Neutropenia cronica maligna familiar con granulaciones atipicas de los leucocitos. Bol Soc Cubana Pediatr 1943:900-22.

42. Chediak M, Chediak B, Fleites O, Hernandez A. [Presentation of a case of Cooley's anemia in a Cuban boy; first report in Cuba.]. Bol Liga Contra Cancer Havana 1952;27:20-6.

43. Chediak MM. [New leukocyte anomaly of constitutional and familial character.]. Rev Hematol 1952;7:362-7.

44. Higashi O. Congenital abnormity of peroxidase granules; a case of congenital gigantism of per- 
oxidase granules, preliminary report. Tohoku J Exp Med 1953;58:246.

45. Barbosa MD, Nguyen QA, Tchernev VT, et al. Identification of the homologous beige and Chediak-Higashi syndrome genes. Nature 1996;382:262-5.

46. Nagle DL, Karim AM, Woolf EA, et al. Identification and mutation analysis of the complete gene for Chediak-Higashi syndrome. Nature Genet 1996;14:307-11.

47. Certain S, Barrat F, Pastural E, et al. Protein truncation test of LYST reveals heterogenous mutations in patients with Chediak-Higashi syndrome. Blood 2000;95:979-83.

48. Karim MA, Suzuki K, Fukai K, et al. Apparent genotype-phenotype correlation in childhood, adolescent, and adult Chediak-Higashi syndrome. Am J Med Genet 2002;108:16-22.

49. Ward DM, Griffiths GM, Stinchcombe JC, Kaplan J. Analysis of the lysosomal storage disease Chediak-Higashi syndrome. Traffic 2000;1:816-22.

50. Tchernev VT, Mansfield TA, Giot L, et al. The Chediak-Higashi protein interacts with SNARE complex and signal transduction proteins. Mol Med 2002;8:56-64.

51. Tardieu M, Lacroix C, Neven B, et al. Progressive neurological dysfunctions twenty years after allogeneic bone-marrow transplantation for Chediak-Higashi syndrome. Blood 2005;106:40-2.

52. Griscelli C, Durandy A, Guy-Grand D, et al. A syndrome associating partial albinism and immunodeficiency. Am J Med 1978;65:691-702.

53. Klein C, Philippe N, Le Deist F, et al. Partial albinism with immunodeficiency (Griscelli syndrome). J Pediatr 1994;125(6 Pt 1):886-95.

54. Pastural E, Barrat FJ, Dufourcq-Lagelouse R, et al. Griscelli disease maps to chromosome 15q21 and is associated with mutations in the myosinVa gene. Nat Genet 1997;16:289-92.

55. Menasche G, Pastural E, Feldmann J, et al. Mutations in RAB27A cause Griscelli syndrome associated with haemophagocytic syndrome. Nat Genet 2000;25:173-6.

56. Menasche G, Ho CH, Sanal O, et al. Griscelli syndrome restricted to hypopigmentation results from a melanophilin defect (GS3) or a MYO5A F-exon deletion (GS1). J Clin Invest 2003;112:450-6.
57. Purtilo DT, Cassel CK, Yang JP, Harper R. $\mathrm{X}$-linked recessive progressive combined variable immunodeficiency (Duncan's disease). Lancet 1975;1:935-40.

58. Chen R, Relouzat F, Roncagalli R, et al. Molecular dissection of 2B4 signaling: implications for signal transduction by SLAM-related receptors. Mol Cell Biol 2004;24:5144-56.

59. Engel P, Eck MJ, Terhorst C. The SAP and SLAM families in immune responses and $\mathrm{X}$-linked lymphoproliferative disease. Nat Rev Immunol 2003;3:813-21.

60. Chan B, Lanyi A, Song HK, et al. SAP couples Fyn to SLAM immune receptors. Nat Cell Biol 2003;5:155-60.

61. Moretta A, Bottino C, Vitale M, et al. Activating receptors and coreceptors involved in human natural killer cell-mediated cytolysis. Annu Rev Immunol 2001;19:197-223.

62. Parolini S, Bottino C, Falco M, et al. X-linked lymphoproliferative disease. 2B4 molecules displaying inhibitory rather than activating function are responsible for the inability of natural killer cells to kill Epstein-Barr virus-infected cells. J Exp Med 2000;192:337-46.

63. Tangye SG, Cherwinski H, Lanier LL, Phillips JH. 2B4-mediated activation of human natural killer cells. Mol Immunol 2000;37:493-501.

64. Tangye SG, Phillips JH, Lanier LL, Nichols KE. Functional requirement for SAP in 2B4-mediated activation of human natural killer cells as revealed by the X-linked lymphoproliferative syndrome. J Immunol 2000;165:2932-6.

65. Silverman ED, Miller JJ 3rd, Bernstein B, Shafai T. Consumption coagulopathy associated with systemic juvenile rheumatoid arthritis. J Pediatr 1983;103:872-6.

66. Hadchouel M, Prieur AM, Griscelli C. Acute hemorrhagic, hepatic, and neurologic manifestations in juvenile rheumatoid arthritis: possible relationship to drugs or infection. J Pediatr 1985;106:561-6.

67. Grom AA. Macrophage activation syndrome and reactive hemophagocytic lymphohistiocytosis: the same entities? Curr Opin Rheumatol 2003;15:587-90.

68. Grom AA, Villanueva J, Lee S, et al. Natural killer cell dysfunction in patients with systemic-onset juvenile rheumatoid arthritis and macrophage activation syndrome. J Pediatr 2003;142:292-6. 
69. Grom AA. Natural killer cell dysfunction: a common pathway in systemic-onset juvenile rheumatoid arthritis, macrophage activation syndrome, and hemophagocytic lymphohistiocytosis? Arthritis Rheum 2004;50:689-98.

70. Normand N, Lehamn T, Elkon K, Onel K. Lower expression of perforin in CD8+ T cells in patients with systemic onset juvenile rheumatoid arthritis [abstract]. Arthritis Rheum 2000;Suppl:S472.

71. Takei M, Ishiwata T, Mitamura K, et al. Decreased expression of signaling lymphocytic-activation molecule-associated protein (SAP) transcripts in $\mathrm{T}$ cells from patients with rheumatoid arthritis. Int Immunol 2001;13:559-65.

72. Risdall RJ, McKenna RW, Nesbit ME, et al. Virus-associated hemophagocytic syndrome: a benign histiocytic proliferation distinct from malignant histiocytosis. Cancer 1979;44:993-1002.

73. Abdelkefi A, Ben Othman T, Torjman L, et al. Plasmodium falciparum causing hemophagocytic syndrome after allogeneic blood stem cell transplantation. Hematol J 2004;5:449-50.

74. Albrecht H, Schafer H, Stellbrink HJ, Greten H. Epstein-Barr virus-associated hemophagocytic syndrome. A cause of fever of unknown origin in human immunodeficiency virus infection. Arch Pathol Lab Med 1997;121:853-8.

75. Auerbach M, Haubenstock A, Soloman G. Systemic babesiosis. Another cause of the hemophagocytic syndrome. Am J Med 1986;80:301-3.

76. Babu TG, Boctor D, Davey A, et al. Cytomegalovirus-associated hemophagocytic syndrome in a child with Crohn disease receiving azathioprine. J Pediatr Gastroenterol Nutr 2004;39:418-21.

77. Banno S, Matsumoto Y, Sugiura Y, Ueda R. [Human parvovirus B19 infection mimicking systemic lupus erythematosus: case report]. Ryumachi 1997;37:581-6.

78. Baraldes MA, Domingo P, Gonzalez MJ, et al. Tuberculosis-associated hemophagocytic syndrome in patients with acquired immunodeficiency syndrome. Arch Intern Med 1998;158:194-5.

79. Bell MD, Wright RK. Fatal virus-associated hemophagocytic syndrome in a young adult producing nontraumatic splenic rupture. J Forensic Sci 1992;37:1407-17.

80. Bird G, Peel D, McCarthy K, Williams H. Epstein-Barr virus induced virus-associated hemophagocytic syndrome and monoclonal TCRbeta rearrangement: a case report. Hematol Oncol 1997; 15:47-52.

81. Blanche P, Robert F, Dupouy-Camet J, Sicard D. Toxoplasmosis-associated hemophagocytic syndrome in a patient with AIDS: diagnosis by the polymerase chain reaction. Clin Infect Dis 1994;19:989-90.

82. Boruchoff SE, Woda BA, Pihan GA, et al. Parvovirus B19-associated hemophagocytic syndrome. Arch Intern Med 1990;150:897-9.

83. Cantero-Hinojosa J, Diez-Ruiz A, Santos-Perez JL et al. Lyme disease associated with hemophagocytic syndrome. Clin Investig 1993;71:620.

84. Chim CS, Fong CY, Ma SK, et al. Reactive hemophagocytic syndrome associated with Penicillium marneffei infection. Am J Med 1998;104:196-7.

85. Chien YH, Lee PI, Huang LM, et al. Typhoid fever presenting as infection-associated hemophagocytic syndrome: report of one case. Acta Paediatr Taiwan 1999;40:339-40.

86. Chien CC, Chiou TJ, Lee MY, et al. Tuberculosis-associated hemophagocytic syndrome in a hemodialysis patient with protracted fever. Int $\mathbf{J}$ Hematol 2004;79:334-6.

87. Real E, Gomez A, Alcaraz MJ, et al. Fulminant hemophagocytic syndrome as presenting feature of T-cell lymphoma and Epstein-Barr virus infection. Haematologica 2000;85:439-40.

88. Ravelli A. Macrophage activation syndrome. Curr Opin Rheumatol 2002;14:548-52.

89. Takasaki N, Kaneko Y, Maseki N, et al. Hemophagocytic syndrome complicating T-cell acute lymphoblastic leukemia with a novel $\mathrm{t}(11 ; 14)(\mathrm{p} 15 ; \mathrm{q} 11)$ chromosome translocation. Cancer 1987;59:424-8.

90. Janka G, Imashuku S, Elinder G, et al. Infectionand malignancy-associated hemophagocytic syndromes. Secondary hemophagocytic lymphohistiocytosis. Hematol Oncol Clin North Am 1998;12:435-44.

91. Su IJ, Hsu YH, Lin MT, et al. Epstein-Barr viruscontaining T-cell lymphoma presents with hemophagocytic syndrome mimicking malignant histiocytosis. Cancer 1993;72:2019-27.

92. Su IJ, Wang CH, Cheng AL, Chen RL. Hemophagocytic syndrome in Epstein-Barr virusassociated T-lymphoproliferative disorders: 
disease spectrum, pathogenesis, and management. Leuk Lymphoma 1995;19:401-6.

93. Lay JD, Chuang SE, Rowe M, Su IJ. Epstein-Barr virus latent membrane protein-1 mediates upregulation of tumor necrosis factor-alpha in EBVinfected T cells: implications for the pathogenesis of hemophagocytic syndrome. J Biomed Sci 2003;10:146-55.

94. Kawaguchi H, Miyashita T, Herbst H, et al. Epstein-Barr virus-infected $\mathrm{T}$ lymphocytes in Epstein-Barr virus-associated hemophagocytic syndrome. J Clin Invest 1993;92:1444-50.

95. Craig FE, Clare CN, Sklar JL, Banks PM. T-cell lymphoma and the virus-associated hemophagocytic syndrome. Am J Clin Pathol 1992;97:189-94.

96. Henter JI, Arico M, Egeler RM, et al. HLH-94: a treatment protocol for hemophagocytic lymphohistiocytosis. HLH study Group of the Histiocyte Society. Med Pediatr Oncol 1997;28:342-7.

97. Caillat-Zucman S, Le Deist F, Haddad E, et al. Impact of HLA matching on outcome of hematopoietic stem cell transplantation in children with inherited diseases: a single-center comparative analysis of genoidentical, haploidentical or unrelated donors. Bone Marrow Transplant 2004;33:1089-95.

98. Arico M, Janka G, Fischer A, et al. Hemophagocytic lymphohistiocytosis. Report of 122 children from the International Registry. FHL Study Group of the Histiocyte Society. Leukemia 1996;10:197-203.

99. Ravelli A, De Benedetti F, Viola S, Martini A. Macrophage activation syndrome in systemic juvenile rheumatoid arthritis successfully treated with cyclosporine. J Pediatr 1996;128:275-8. 